\title{
Intelligent Deviation Perception and Prediction of Deviation Probability
}

\author{
O. Birth ${ }^{1,2}$ and F. Yu ${ }^{2}$ \\ ${ }^{1}$ Connected Drive Department, BMW Group Research and Technology, 80992 Munich, Germany \\ ${ }^{2}$ Institute of Applied Informatics \& Cooperative Systems, Technische Universitaet Muenchen, \\ 85748 Garching, Germany
}

Keywords: deviation prediction, dimension, logic

\begin{abstract}
Innovation article about architecture of deviation decision, which expands a deviation perception into three dimensions as time, space and logic, and externalizes the existence as well as the degree of a potential deviation in form of probability. To develop deviation into logic dimension, several common logical reasons concerning daily scenario are classified as positive, negative and neutral reasoning, and corresponding formulas are given, to either support or dispute a potential deviation suggested by the dimensions as time and space.
\end{abstract}

\section{Introduction}

Deviation is common in daily life. In certain degree, deviations are supposed to be tolerated. People hardly care whether the GPS-position has a deviation of two-meter, or the subway delayed by 30s while one still managed to get the next train, nevertheless, when the GPS-position has a deviation of 200 meter or the punctuality of the public transport enabled the one not be able to catch up with the next train, information about deviations become important. In the presented example, the deviation infers that a person not only deviated in time and space, but also drops out the appointment one intends to overtake.

Besides, the recognition of the user intention is currently difficult. Several study in artificial intelligence and related fields have already shown the possibility to deduce the intention of humankind [1],[2]. However, before highly precise methods of intention recognition are established, one of the most practical approach to speculate whether one gets lost is to compare the outcome with the given target, and then search for logical reasoning in periphery environment to explain the potential deviation. Whether the reasoning process failed to dispute the potential deviation, a deviation can be ascertained. It's a deviation in logic. Therefore, when one complains about delay, which is actually a negative deviation in scientific sense, it's equivalent to talk about the deviation in dimensions as time, space, logic, or the combination of the terms. In order to measure the existence and degree of a deviation, the quantification of the deviation is necessary for subsequent research, which can be achieved by the calculation of corresponding probability. This paper focuses on the attempt to externalize the deviation in mathematical form, and present the existence and degree of a deviation in dimensions as time, space, and logic in probability.

\section{Related Research}

Concerning GPS-based navigation system, measuring the distance between user and planned route is continuously the main method, to find the existence of deviation. However, there're increasing study on the relationship between deviation and expected travel time. Recent researches suggest, that travel time prediction can be carried out by means of using the historical movement data, and the predicted travel time can be achieved in form of distribution [3]. Related work also shows the possibility of predicting the travel time by applying the Kalman filtering and estimation technique in real-time [4]. 
To further predict the user's future movement, there's a method suggesting the clustering of GPS data over certain time at multiple scales, and a Markov model is introduced by processing the corresponding locations where the GPS data are taken [5].

Nevertheless, in comparison to deviation decision based on the measurement of distance, more studies on revealing the relation between deviation and time are expected. While there're fewer researches on deviation decision in logic dimension, and the user's motion behaviour in the past is hardly taken into consideration. Moreover, the prediction of a potential deviation is a fairly new topic in the current research field.

\section{Hypotheses}

A deviation is the divergence between expected set point and actual value, which can be regarded as an integrated outcome synthesized by dimensions in time, space, and logic level. The degree of deviation signifies the extent of possibility, how the expected target is managed to be satisfied. Before introducing the methodology, several hypotheses are proposed, in order to predigest the study and set up ideal model.

\section{Hypothesis I: Rationality}

User is rational. The user is supposed to set up start position as well as target position at the very beginning, and always moves according to the suggested route approximately. Though it can possibly be that the user is not aware of the potential deviation, whenever there exists a deviation of large scale, the deviation is expected to be discovered by the user, and the user would attempt to correct it in an appropriate way.

\section{Hypothesis II: Finite Purposes}

User has limited purposes. Based on the hypothesis I, the user's ultimate final target is regarded in vicinity of the target position. Because of car parking, running into acquaintance etc., or user didn't set the target position accurately, fairish target range is to be tolerated. The prediction phase works under this hypothesis.

\section{Hypothesis III: Regularity}

User is expected to have regular motion behaviour. The walking speed (in walking mode), fuzzy maximum deviation distance, fuzzy maximum deviation time are expected to be maintained as stable values, so that minor fluctuation and oscillation could be regarded as obeying statistical laws.

\section{General Description of the Methodology}

Deviation can be regarded as a complex constituted by three dimensions: deviation in time, in space, and in logic .The perception process of the deviation detection is considered as black-box-system, with potential deviation in all dimensions as input, surrounding environment and user historical motion behaviour as reference basis, and entire probability of deviation as output. To deduce and simulate the internal principle of the box as precisely as possible, it can be convert to the architecture shown in Fig. 1.

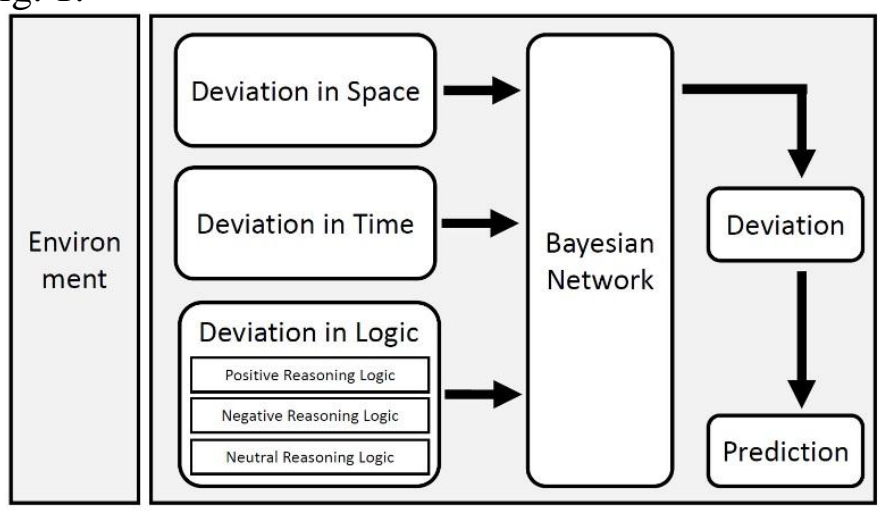

Fig. 1: Overview of the architecture

Deviation Perception via Bayesian Network 
The overall perception of deviation is performed by Bayesian network. Since a potential deviation is divided into three dimensions: space, time and logic, the degree of potential deviation in space dimension, in time dimension, and in logic dimension are supposed to be summed up to form a deviation. Denoting a perception as $Y$, perception of deviation and non-deviation as $Y=1$ and $Y=0$ respectively, the input of the Bayesian network are:

Given a perception of deviation in space as $x=\theta$, the probability of deviation in space dimension based on historical distribution are:

$$
P_{(x \leq \theta \mid Y=1)}, P_{(x \leq \theta \mid Y=0)}
$$

Given a perception of deviation in time as $x=\varphi$, the probability of deviation in time dimension based on historical distribution:

$$
P_{(x \leq \varphi \mid Y=1)}, P_{(x \leq \varphi \mid Y=0)}
$$

Given a perception of deviation in logic as $x=\omega$, the probability of deviation in logic dimension based on historical distribution:

$$
P_{(x \ll \omega \mid Y=1)}, P_{(x \ll \omega \mid Y=0)}
$$

The probability of perception of deviation is:

$$
\mathrm{P}(\mathrm{x} ; \theta, \varphi, \omega \mid \mathrm{Y}=1)=P_{(x \leq \theta \mid Y=1)} \cdot P_{(x \leq \varphi \mid Y=1)} \cdot P_{(x<\omega \mid Y=1)} \cdot P(Y=1)
$$

While the probability of perception of non-deviation is:

$$
\mathrm{P}(\mathrm{x} ; \theta, \varphi, \omega \mid \mathrm{Y}=0)=P_{(x \leq \theta \mid Y=0)} \cdot P_{(x \leq \varphi \mid Y=0)} \cdot P_{(x \ll \omega \mid Y=0)} \cdot P(Y=0)
$$

The perception of deviation can be performed by comparing $P(x ; \theta, \varphi, \omega \mid Y=1)$ and $\mathrm{P}(\mathrm{x} ; \theta, \varphi, \omega \mid \mathrm{Y}=0)$.

\section{Deviation in Space Dimension}

As described above, we will first describe how to detect deviation in the space dimension. The actual position of an user is acquired via GPS raw data in real time. Regardless of deviation in the other dimensions, several rules are to be formulated independently and respected, to build up certain criterion measuring the extent of deviation in space. In principle, the farther the distance between user's current location and suggested route is, the higher the probability should be, that the user deviates. The deviated distance should be weighted distinctly to the total travelling distance, which means the probability of a deviation in space should be dynamic. Every outcome of the whole deviation perception and the deviation in space are able to be retrieved from historical list, and contribute to further processes and iterations.

\section{Calculation of the Probability of Deviation in Space Dimension}

Denoting the probability of deviation in space as $P_{(x=\theta)}$, the whole travelling distance and potential deviated distance as $\mathrm{L}$ and $\theta$ respectively, the calculation of deviation in space dimension is performed as follows:

$$
P_{(x=\theta)}=-\left(\frac{1}{2}\right)^{\frac{\pi^{2} \theta}{L}}+1
$$

Denoting an outcome of potential deviation perception in the past as set $\{Y\}$, and $Y=1$ as a deviation, $\sum n$ as number of perception $|\{Y\}|, n_{0}$ and $n_{1}$ as number of non-deviation $|\{Y=0\}|$ and deviation $|\{Y=1\}|$ respectively. Because of the incompatibility, it satisfies:

$$
\sum \mathrm{n}=|\{Y\}|=|\{Y=0\}|+|\{Y=1\}|=\mathrm{n}_{0}+\mathrm{n}_{1}
$$

In which, given a potential deviation in $\operatorname{space} P_{\left(x=\theta_{0}\right)},\{Y=1\}$ can further be divided as $\{Y=1\}=$ $\left\{\mathrm{x} \mid \mathrm{x} \in\left[0, \theta_{0}\right], \mathrm{Y}=1\right\} \cup\left\{\mathrm{x} \mid \mathrm{x} \in\left(\theta_{0},+\infty\right), \mathrm{Y}=1\right\}$.

Therefore, the probability of $P_{\left(x \leq \theta_{0} \mid Y=1\right)}$ and $P_{\left(x \leq \theta_{0} \mid Y=0\right)}$ can be expressed as follows:

$$
\begin{aligned}
& P_{\left(x \leq \theta_{0} \mid Y=1\right)}=\frac{\mathrm{P}\left(\mathrm{Y}=1 \mid \mathrm{x} \ll \theta_{0}\right) \cdot \mathrm{P}\left(\mathrm{x} \ll \theta_{0}\right)}{\mathrm{P}(\mathrm{Y}=1)}=\frac{\left|\left\{\mathrm{x} \mid \mathrm{x} \in\left[0, \theta_{0}\right], \mathrm{Y}=1\right\}\right|}{\mathrm{n}_{1}}, \\
& P_{\left(x \leq \theta_{0} \mid Y=0\right)}=\frac{\mathrm{P}\left(\mathrm{Y}=1 \mid \mathrm{x} \ll \theta_{0}\right) \cdot \mathrm{P}\left(\mathrm{x} \ll \theta_{0}\right)}{\mathrm{P}(\mathrm{Y}=0)}=\frac{\left|\left\{\mathrm{x} \mid \mathrm{x} \in\left[0, \theta_{0}\right], \mathrm{Y}=0\right\}\right|}{\mathrm{n}_{0}} .
\end{aligned}
$$

Monotonicity

Taken derivative of the function $P_{(x=\theta)}$, 


$$
\frac{\mathrm{d} P_{(x=\theta)}}{\mathrm{dx}}=\frac{\mathrm{d}\left(-\left(\frac{1}{2}\right)^{\frac{\pi^{2} \theta}{L}}\right)+1}{\mathrm{~d} \theta}=-\frac{\pi^{2}}{\mathrm{~L}} \ln \left(\frac{1}{2}\right)\left(\frac{1}{2}\right)^{\frac{\pi^{2} \theta}{L}} \geq 0, \theta \in[0,+\infty),
$$

the function monotonically increases on domain $[0,+\infty)$ and has a range of $[0,1]$. Since the function has inverse function on domain $[0,1]$, the domain and range of the function has one-to-one correspondence.

\section{Convexity}

Considering the $\frac{\theta}{\mathrm{L}}$ as independent variable, the convex function always has $\mathrm{f}\left(\frac{\mathrm{x}_{1}+\mathrm{x}_{2}}{2}\right)>\frac{\mathrm{f}\left(\mathrm{x}_{1}\right)+\mathrm{f}\left(\mathrm{x}_{2}\right)}{2}$ on domain $[0,1]$. Since the function has $P_{(x)}=x, \mathrm{x}_{1}=0$, and $P_{(x)}>x, \mathrm{x}_{2}=1, P_{(x)}-x \geq 0$ always satisfies on domain $[0,1]$.

\section{Increment of Efficiency via Gaussian distribution}

In order to achieve high accuracy of probability calculation, a great amount of user historical data are required, which is negative to system efficiency and storage capacity. For every time, the user motion is independent of other motions, and every motion can be regarded as random input, Therefore, based on the hypothesis III, the distribution of probability of deviation in space dimension can be assumed as Gaussian distribution.

For a Gaussian distribution $x \sim N\left(\mu, \sigma^{2}\right)$, the distribution can be completely formed by two parameters $\mu$ and $\sigma^{2}$ :

$$
\begin{aligned}
& \mu_{0}=\frac{\sum \mathrm{x}}{\mathrm{n}_{0}}, \mathrm{x} \in\{\mathrm{x} \mid \mathrm{Y}=0\}, \sigma_{0}{ }^{2}=\frac{\sum(x-\bar{x})^{2}}{n_{0}}, \mathrm{x} \in\{\mathrm{x} \mid \mathrm{Y}=0\}, \\
& \mu_{1}=\frac{\sum \mathrm{x}}{\mathrm{n}_{1}}, \mathrm{x} \in\{\mathrm{x} \mid \mathrm{Y}=1\}, \sigma_{1}{ }^{2}=\frac{\sum(x-x)^{2}}{n_{1}}, \mathrm{x} \in\{\mathrm{x} \mid \mathrm{Y}=1\},
\end{aligned}
$$

When a new perception of potential deviation in space dimension $\mathrm{x}_{\mathrm{t}}=P_{\left(\mathrm{x}_{\mathrm{t}}=\theta\right)}$ occurs, the update of user historical data can be performed as follows:

$$
\begin{aligned}
& \mu_{0}^{\prime}=\frac{\sum \mathrm{x}^{\prime}}{\mathrm{n}_{0}^{\prime}}=\frac{\sum \mathrm{x}+\mathrm{x}_{\mathrm{t}}}{\mathrm{n}_{0}^{\prime}}=\frac{\mu_{0} \mathrm{n}_{0}+\mathrm{x}_{\mathrm{t}}}{\mathrm{n}_{0}+1}, \sigma_{0}^{\prime 2}=\frac{\sum\left(x-\bar{x}^{\prime}\right)^{2}}{\mathrm{n}_{0}^{\prime}}=\frac{\sum\left(x-\bar{x}^{\prime}\right)^{2}}{\mathrm{n}_{0}+1}, \\
& \mu_{1}^{\prime}=\frac{\sum \mathrm{x}^{\prime}}{\mathrm{n}_{1}^{\prime}}=\frac{\sum \mathrm{x}+\mathrm{x}_{\mathrm{t}}}{\mathrm{n}_{1}^{\prime}}=\frac{\mu_{1} \mathrm{n}_{1}+\mathrm{x}_{\mathrm{t}}}{\mathrm{n}_{1}+1}, \sigma_{1}^{\prime 2}=\frac{\sum\left(x-\bar{x}^{\prime}\right)^{2}}{\mathrm{n}_{1}^{\prime}}=\frac{\sum\left(x-\bar{x}^{\prime}\right)^{2}}{\mathrm{n}_{1}+1},
\end{aligned}
$$

With the assumption of Gaussian distribution, the user historical data can be managed via two key parameters and updated in a straightforward way. Moreover, the system is supposed to detect a potential deviation in early time. When a user already deviates $\frac{\theta}{\mathrm{L}} \gg 0.5$, it's nearly nothing different to deviate 0.7 or 0.6 . Hence, the function should be fairly sensitive to an early time potential deviation, which means $P_{(x=\theta)}$ is expected to be weighted more in early time. In this case, a convex function can be a reasonable choice, and because of the reasons above, a function possessing monotonicity and convexity is selected as an ideal transformation of independent variable for Gaussian distribution.

\section{Deviation in Time dimension}

With certain time interval, the rest of travelling time to the target position is estimated. The deviation in time comes actually from two parts, whether the user is behind of the regular estimated travelling time point, and the time user is diverged from the normal travelling route. To construct proper regulation assessing the deviation in time, several rules are established. In case that the estimated travelling time at a certain position in real time is beyond certain distance, which is dynamically suggested by the original travelling time from start position, the probability that user deviates in time increases. At the same moment the vertical distance between user and suggested route is over a dynamically determined threshold, a timer awakes to count the potential deviation time. The threshold deals with the inaccuracy of GPS-positioning. Moreover, it also serves for the problem, when people walks on the sidewalk. Not like the cars must drive on the right in many countries, people are usually able to feel free to walk on either side of sidewalk, therefore, it can be a source of error compared to the standard location data point suggested by route planning. 
Denoting the whole travelling time as $\sum T$, estimated travelling time as $T_{E s t}$ and actual travelling time as $T_{A c t}$, the criterion of high deviation in time is:

Which is equivalent to,

$$
\frac{T_{E s t}-T_{A c t}}{\sum T} \gg\left(\frac{T_{A c t}}{\sum T}\right)^{2}
$$

$$
T_{E s t} \gg \frac{T_{A c t}^{2}}{\sum T}+T_{A c t}
$$

The probability of deviation in time dimension $P_{(x=\varphi)}$ is achieved by the following function, where the $\varphi$ stands for potential deviation time.

$$
P_{(x=\varphi)}=\frac{e^{\frac{\varphi}{\sum T}}-e^{-\frac{\varphi}{\sum T}}}{e^{\frac{\varphi}{\sum T}}+e^{-\frac{\varphi}{\sum T}}}
$$

Similarly to the potential deviation in space dimension, $P_{(x \leq \varphi \mid Y=1)}$ as well as $P_{(x \leq \varphi \mid Y=0)}$ can be calculated through Gaussian distribution.

In view of the hypothesis I, when the user attempts to come back to the suggested route, it should be looked upon as implication of being aware of deviation, which is forcefully a good signal of nondeviation.

For every coming back to the suggested route $\mathrm{k}$, the potential deviated time $\varphi$ is regressed to $\varphi_{k}$ as follows, where $P_{(\omega)}$ implies the probability of deviation in logic dimension discussed in chapter [3.3]:

$$
\begin{aligned}
\varphi_{1} & =\prod \mathrm{P}\left(\mathrm{x}_{\mathrm{t}} ; \theta, \varphi, \omega \mid \mathrm{Y}=1\right)^{0} \cdot \varphi_{0} \\
\varphi_{2} & =\prod \mathrm{P}\left(\mathrm{x}_{\mathrm{t}} ; \theta, \varphi, \omega \mid \mathrm{Y}=1\right)^{1} \cdot \varphi_{1} \\
\varphi_{k} & =\prod \mathrm{P}\left(\mathrm{x}_{\mathrm{t}} ; \theta, \varphi, \omega \mid \mathrm{Y}=1\right)^{k-1} \varphi_{k-1} \\
\varphi_{1} \varphi_{2} \cdots \varphi_{k}= & \prod \mathrm{P}\left(\mathrm{x}_{\mathrm{t}} ; \theta, \varphi, \omega \mid \mathrm{Y}=1\right)^{0+1+\cdots+k-1} \varphi_{0} \varphi_{1} \cdots \varphi_{k-1} \\
\varphi_{k} & =\varphi_{0} \prod \mathrm{P}\left(\mathrm{x}_{\mathrm{t}} ; \theta, \varphi, \omega \mid \mathrm{Y}=1\right)^{\frac{k(k-1)}{2}}
\end{aligned}
$$

\section{Deviation in Logic dimension}

Though the user approximately moves on the basis of the planned route, it still doesn't necessarily mean that the user has no risk of being deviated. Because of the existing road planning, if the user makes an inappropriate decision on crossroad, it can possibly leads to a deviation in this situation, although the user is still very near the planned route. Apparently the user produces deviation neither in time nor in space, but in logic. In this situation, the deviation in logic appears and remains, and leads to the result that the deviation in both time and space are increasing over time. Deviation in logic not only helps refute a potential deviation which is suggested by any other dimensions, but also can figure out the eventuality of deviation risk, while the potential deviation is not obvious yet. The entire deviation in logic dimension is consist of positive, negative and neutral logical reasoning part.

These logical reasoning components above either refute or sustain a potential deviation suggested by the other dimensions. The outcome of these reasoning parts will be synthesized according to the Bayesian network as an epitomized probability of deviation in logical dimension $P_{(x=\omega)}$.

\section{Classification of Logical Reasoning Criterion}

A potential deviation in logic dimension can be divided into three classes: positive logical reasoning, negative logical reasoning and neutral logical reasoning.

An example for a typical negative logical reasoning is the divergence between set point and actual position. Commonly speaking, according to the suggested plan, the user is expected to be at position $p_{0}$ at moment $t_{0}$, while actually the user is at position $p_{0}^{\prime}$. When the divergence between $p_{0}$ 
and $p_{0}^{\prime}$ is slight, the difference can possibly be tolerated. However, when the divergence is of great value, the user should think about whether he/she can get to target in planned time. In this meaning, the probability of potential deviation is positively correlated with divergence, and hence, the divergence supports the existence of deviation. It can be classified as negative logical reasoning, and the mean or expectation of Gaussian distribution is usually of high value, when there exists a deviation.

On the other hand, when there's a negative correlation between independent variable and dependent variable, it is classified as positive logical reasoning. For example, a user is near his/her favourite restaurant, and the greater the probability is, that the user is tend to visit it, the smaller the probability is, that the user deviates. In a words, the increment of input helps refute the existence of deviation, and so that it is classified as positive logical reasoning. Its mean or expectation of Gaussian distribution is normally small value, when a deviation is observed. When there's no clear tendency whether it's a positive or negative correlation between the independent variable and dependent variable, it's classified as neutral logical reasoning.

\section{Positive Logical Reasoning}

The positive logical reasoning can be applied in vicinity of the target position and visited facilities nearby.

\section{In vicinity of the target position}

There are numbers of possibilities and situations that it seems the user deviates, while it's not indeed, e.g. when user is looking for a parking slot, the user usually looks like that he/she lost the way in a while, the user leaves the suggested route for a certain time and distance to check if there's a parking slot nearby; or a user walks around in a circle to find a suitable slot, which is commonly regarded as a clue of deviation.

In this meaning, although the probability of the deviation has a quite high value, the deviation in logic is responsible for disputing the potential deviation.

Since the user's motion behaviour depends on the weight of distance to target location $L_{r}$, and measuring whether the user is nearly to target is equivalent to measuring the distance to the starting point, and a activation threshold should be set to ensure, that the user is in certain distance to the target. The calculation can simply be:

$$
P_{\left(i_{0}\right)}=\mathrm{C} \frac{L-L_{r}}{L}, \mathrm{~L}_{\mathrm{r}} \in(0, \mathrm{R})
$$

where $C$ is the relaxation coefficient, and $\mathrm{R}$ is the activation threshold. Therefore, when the $P_{\left(i_{0}\right)}$ has a high value, it usually implies that the user is in vicinity of the target.

\section{Visited facilities nearby}

Another scenario in daily life is, one walks or drives towards a target through a places or facility, which he/she usually visited. Then the user changes the mind, to visit for a while for reasons like shopping, having a coffee etc., which can generally be tolerated from a potential deviation. Nevertheless, when the user detours in direction of these facilities but passed by, which means the user does not intend to visit, the possibility of visiting is to be excluded.

The distance denoted as $L_{n} \in\{L\}, n \in N$ between the user and facilities plays a prominent role, while estimating the chance if a user tends to go there. Distances larger than $l_{L}$ denoted as $L_{T}$ are regarded as too far away and will be excluded. Distances smaller than $l_{L}$ can be divided into $L_{L}, L_{M}$, and $D_{H}$, which represent the distances of low, medium and high probability respectively. Therefore, a division can be:

$$
L_{n} \in\{L\}=\left\{L_{H}\right\} \cup\left\{L_{M}\right\} \cup\left\{L_{L}\right\} \cup\left\{L_{T}\right\}=\left[0, l_{H}\right)\left[l_{H}, l_{M}\right)\left[l_{M}, l_{L}\right]\left(l_{L},+\infty\right)
$$

A classifier dealing with highly reachable distances can be:

$$
L_{n} \in\left\{L_{H}\right\} \cup\left\{L_{M}\right\} \cup\left\{L_{L}\right\}=\left[0, l_{H}\right)\left[l_{H}, l_{M}\right)\left[l_{M}, l_{L}\right] \subsetneq\{L\}
$$

Denoting whether a facility was visited at last as $V_{1}, V_{0}$, the initial probabilities a user inclines to visit the facility with different distances are denoted as follows and to be kept updating with every new visiting:

$$
P\left(L_{H} \mid V_{1}\right), P\left(L_{H} \mid V_{0}\right), P\left(L_{M} \mid V_{1}\right), P\left(L_{M} \mid V_{0}\right), P\left(L_{L} \mid V_{1}\right), P\left(L_{L} \mid V_{0}\right)
$$


To specify if a user is visiting a facility, user motion is tracked and identified by the fact whether the user stops at a position nearby, which is denoted as $S_{1}$. Furthermore, passing by denoted as $S_{0}$ is identified as no desire to visit and has high probability $P\left(x=S_{0} \mid Y=0\right)$ that the user is lost, for the other case $P\left(x=S_{0} \mid Y=1\right)$. By every passing by of the facilities $P\left(x=S_{0} \mid Y=1\right)$, the probability of information gain $P_{\left(i_{1}\right)}$ is given by following function, where the number of facilities nearby is denoted as $n$ :

\section{Negative Logical Reasoning}

$$
P_{\left(i_{1}\right)}=\left(\frac{1}{\pi}\right)^{\sqrt{\sum_{i=0}^{n} P_{\left(s_{1}\right)}}}
$$

In many situations, logic also figures out a deviation vice versa, although the deviation in time and space looks fine. Whenever the user is behind the timing, there's divergence between the planned location and actual location, which indicates a negative deviation. In contrast, a positive deviation sufficiently involves, that user is before timing and has therefore enough time to deal with latent mistakes or deviations. The probability of the deviation between set point and actual location can be calculated as follows:

$$
P_{\left(i_{2}\right)}=-\left(\frac{1}{2}\right)^{\frac{e \tau}{s}}+1
$$

Another indicator for a deviation is abnormal motion alteration, e.g. user stops moving or moves backwards frequently, which are commonly deemed as behaviours during getting lost. However, because there's no continuity in these user behaviours, the probability of abnormal motion alteration cannot be calculated like the other probability of deviation in logic dimension. Therefore, the calculation is able to be performed by introducing the Poisson distribution.

The frequency that user stops $N_{S}$ or moves backwards $N_{B}$ and in certain time period T obeys Poisson distribution $P(X=i)=\frac{e^{-\lambda} \lambda^{i}}{i !}, i \in N$, where the $\lambda$ is the expected value as well as variance.

The probability the user arrives the goal position in time, while stops or moves back on the deviation road, $P\left(x=N_{S}\right)=\frac{e^{-\lambda_{1}} \lambda_{1}{ }^{i}}{i !}, P\left(x=N_{B}\right)=\frac{e^{-\lambda_{2} \lambda_{2}}{ }^{i}}{i !}$, and $P\left(i_{3}\right)$ is the weighted average of $P\left(x=N_{S}\right)$ and $P\left(x=N_{B}\right)$.

Therefore, $P\left(x \ll N_{S}\right)$ and $P\left(x \ll N_{B}\right)$ can be achieved from Poisson CDF.

\section{Neutral Logical Reasoning}

There're many examples in daily life, that one's decision actually increases the risk of deviation, while one is still quite close to the suggested route, e.g. at the crossroad. User's motion behaviours act differently at the standpoint, in comparison to the suggested one. This comparison can be acquired by the velocity vector calculation.

With the help of velocity vectors, the user's motion can be tracked and analysed, so that a potential deviation in logic can be found in early time. The velocity vectors calculation serves for reasoning the user's motion behaviours.

Given an actual point $\mathrm{P}_{1}\left(\mathrm{x}_{1}, \mathrm{y}_{1}\right)$, and a point of last instant $\mathrm{P}_{0}\left(\mathrm{x}_{0}, \mathrm{y}_{0}\right)$, the velocity vector of actual motion can be denoted as $\vec{A}=\left(x_{1}-x_{0}, y_{1}-y_{0}\right)$. Acquired from suggested route, velocity vector of expected motion can be denoted as $\vec{B}=\left(x_{1}^{*}-x_{0}^{*}, y_{1}^{*}-y_{0}^{*}\right)$. The dot product of both velocity vectors can be calculated as: $u=\cos (\theta)=\frac{\vec{A} \cdot \vec{B}}{|\vec{A}| \cdot|\vec{B}|}=\frac{\left(x_{1}-x_{0}, y_{1}-y_{0}\right) \cdot\left(x_{1}^{*}-x_{0}^{*}, y_{1}^{*}-y_{0}^{*}\right)}{\sqrt{\left(x_{1}-x_{0}\right)^{2}+\left(y_{1}-y_{0}\right)^{2}} \cdot \sqrt{\left.\left(x_{1}^{*}-x_{0}^{*}\right)^{2}+y_{1}^{*}-y_{0}^{*}\right)^{2}}}$

Since $\cos (2 \mathrm{k} \pi)=1, \mathrm{k} \in \mathrm{Z}$ which implies an ideal motion that user moves completely in right direction suggested by planned route. Whenever there exists any angle $\theta$ between the user moving direction and the suggested one, 
$\cos (2 \mathrm{k} \pi)-\cos (\theta)=-2 \sin \left(\frac{2 k \pi+\theta}{2}\right) \sin \left(\frac{2 k \pi-\theta}{2}\right)=-2 \sin \left(k \pi+\frac{\theta}{2}\right) \sin \left(k \pi-\frac{\theta}{2}\right)=$ $2 \sin \left(\frac{\theta}{2}\right)^{2} \gg 0$.

The probability deduced from velocity vector can be calculated through divergence between actual moving direction and suggested one by cumulating the between angles as:

Denoting the user moving time as $t$,

$$
P_{\left(i_{4}\right)}=\frac{\sum_{i=0}^{t}(\cos (2 \mathrm{k} \pi)-\mathrm{u})}{t}=\frac{\sum_{i=0}^{t}\left(\cos (2 \mathrm{k} \pi)-\cos \left(\theta_{t}\right)\right)}{t}=\frac{\sum_{i=0}^{t} 2 \sin \left(\frac{\theta_{t}}{2}\right)^{2}}{t}
$$

\section{Conclusions}

This paper provides a preliminary approach of study in deviation perception, by dividing a potential deviation in space, time and logic dimension. It focuses on exploring a new architecture of deviation decision, by means of synthesizing potential deviation in all dimensions. Moreover, the degree of deviation in each dimension can be obtained by calculation of their probability, which converses the measurement of deviation in certain dimension into mathematical solution, and corresponding formulas are suggested. For improvement of efficiency, assumption of Gaussian distribution is adopted. To further describe the processing of deviation in logic dimension, several real life scenarios are introduced and given as examples of positive, negative and neutral reasoning respectively.

The theory was tested with Android 4.3 in Munich, and results show sufficient sensitivity to potential deviation concluded by all of the three dimensions, and certain reasoning ability of deviation in logic with real-life scenario, e.g. frequently visited facilities nearby, divergence between expected position and actual one etc. Besides, after tester is aware of the deviation, countermeasures are taken in corresponding situations, e.g. coming back to the planned route, or quickening the pace etc, the architecture presents required adaptivity and tolerance.

Next, we will be acquiring the entire probability of deviation in logic via reasoning based multilayer neural network, whose initial weights are suggested by distribution of reasoning neurons. We will further perform prediction of a deviation with double exponential smoothing. In addition to the classical double ES, a dynamic smoothing factor selection will be suggested, to make the prediction phase more adaptive and flexible to real-time environment.

\section{References}

[1] Korb, K. \& Nicholson, A. E. Bayesian Artificial Intelligence. Chapman \& Hall/CRC, 2003

[2] Murphy, K. P., Dynamic Bayesian Networks. Probabilistic Graphical Models (M. Jordan ed.), 2002

[3] Mbiydzenyuy, G. et al, Road Travel Time Prediction - A Micro-Level Sampling Approach, Sch. of Comput., Blekinge Inst. of Technol., Karlshamn, Sweden, 2013

[4] Jiann-Shiou Y., Travel Time Prediction Using The GPS Test Vehicle And Kalman Filtering Techniques, Dept. of Electr. \& Comput. Eng., Minnesota Univ., Duluth, MN, USA, 2005

[5] Ashbrook, D. et al, Learning Significant Locations And Predicting User Movement With GPS, Coll. of Comput., Georgia Inst. of Technol., Atlanta, GA, USA, 2002 